\title{
Boundary effects of a nonconcentric semipermeable sphere using Happel and Kuwabara cell models
}

\author{
M. Krishna Prasad ${ }^{a, *}$ \\ ${ }^{a}$ Department of Mathematics, National Institute of Technology, Raipur-492010, Chhatisgarh, India \\ Received 20 May 2020; accepted 20 January 2021
}

\begin{abstract}
The effect of a closed boundary on the hydrodynamic drag of a nonconcentric semipermeable sphere in an incompressible viscous fluid is investigated. Darcy's law holds in the permeable region and Stokes flow used inside the spherical cavity. Suitable boundary conditions are used on the surface of a semipermeable sphere and spherical cavity. Two spherical coordinate systems are used to solve the problem. By superposition principle, a general solution is constructed from the solutions based on the semipermeable sphere and spherical cavity. Numerical results for the hydrodynamic drag force exerted on the particle is obtained with good convergence for various values of the relative distance between the centers of the inner sphere and spherical cavity, permeability parameter and the separation parameter. The numerical values of the hydrodynamic drag force generalize the results obtained for an eccentric solid sphere.
\end{abstract}

(C) 2021 University of West Bohemia. All rights reserved.

Keywords: semipermeable sphere, Stokes flow, Darcy’s law, nonconcentric, drag

\section{Introduction}

The low velocity flow around an object like solid, semipermeable, permeable or composite particles has been interesting subject in several scientific and engineering applications exemplified pellets as catalysts, fluid-solid disperse systems, sedimentation etc. Many investigators over the world carried out researches on numerous problems concerning flow through porous bodies. Leonov [16] examined the porous sphere by a uniform stream of an incompressible viscous fluid. Joseph and Tao [9] discussed the coupled problem of a viscous fluid past a permeable sphere. Feng and Michaelides [3] obtained the drag force exerted on permeable sphere at finite but small Reynolds numbers. Srinivasacharya [18] studied the Stokes flow past a porous approximate spherical shell using Darcy's law. Shapovalov [17] discussed the highly viscous flow past a partially permeable spherical particle. Vereshchagin and Dolgushev [20] studied the filtration flow through the spherical shell. Yadav et al. [21,22] investigated the hydrodynamic permeability of membranes built up by porous spherical shells, and porous deformed spheroidal particles using particle-in-cell method.

Gluckman et al. [6] proposed boundary collocation method for slow viscous flow past a finite assemblage of particles. Using this multipole truncation technique, many researchers solved multi-particle interaction, particle-wall boundaries, and non-concentrated problems (Leichtberg et al. [15], Ganatos et al. [4,5], Dagan [1], Keh and Lee [10]). Faltas and Saad [2] investigated the slow motion of slip sphere in an eccentric cell using Happel and Kuwabara boundary conditions [7, 14]. Srivastava et al. [19] studied the flow of an incompressible viscous fluid

\footnotetext{
*Corresponding author. E-mail: madaspra.maths@nitrr.ac.in, kpm973@gmail.com. https://doi.org/10.24132/acm.2021.620
} 
past a porous sphere in presence of transverse applied uniform magnetic field using particle-incell method. Recently, Krishna Prasad and Bucha [13] studied the flow past a semipermeable sphere under magnetic effect. Krishna Prasad [12] investigated the micropolar fluid past a semipermeable sphere using different cell models. Yadav et al. [23] studied the hydrodynamic permeability of a porous spheroidal particles. Khanukaeva et al. [11] examined the creeping flow of micropolar fluid parallel to the axis of porous cylindrical cells. Yadav et al. [24] investigated motion of a non-homogeneous porous spherical particle in a spherical container.

In present paper, we have extended the work done by Shapovalov [17] to an eccentric semipermeable sphere in cell models. The hydrodynamic resisting force acting on the particle is obtained. Numerical results for the case of motion of solid and semipermeable particle in a concentric of closed boundary are included.

\section{Mathematical statement}

Consider the slow motion of a semipermeable spherically symmetric particle with radius $a$ in an incompressible viscous fluid, surrounded by a non-concentric spherical cavity with radius $b$, as depicted in Fig. 1. Let $\eta=\frac{a}{b}$ be the separation parameter. The spherical cavity is frictionless. The fluid comes near the cavity surface and past a semipermeable sphere translating at a constant velocity $U$ from the negative $z$-axis. Here, $(r, \theta, \phi)$ and $(\rho, \phi, z)$ denote the spherical coordinate and circular cylindrical systems, respectively. The origin is fixed at the centre of spherical cavity. The centre of the semipermeable sphere is placed at a distance $d$ from the centre of the cavity. Let $\left(r_{1}, \theta_{1}, \phi\right)$ and $\left(r_{2}, \theta_{2}, \phi\right)$ be the spherical coordinates based on the centre of a semipermeable sphere and spherical cavity, respectively. The relation between the radii of semipermeable sphere $r_{1}$ and spherical cavity $r_{2}$ is given by $r_{1}^{2}=r_{2}^{2}+d^{2}-2 r_{2} d \cos \theta_{2}$ or $r_{2}^{2}=r_{1}^{2}+d^{2}+2 r_{1} d \cos \theta_{1}$.

The field equations governing the flow outside the semipermeable sphere are the continuity and Stokes equations

$$
\begin{aligned}
\nabla \cdot \vec{v}^{(1)} & =0, \\
\nabla p^{(1)}+\mu \nabla \times \nabla \times \vec{v}^{(1)} & =0 .
\end{aligned}
$$

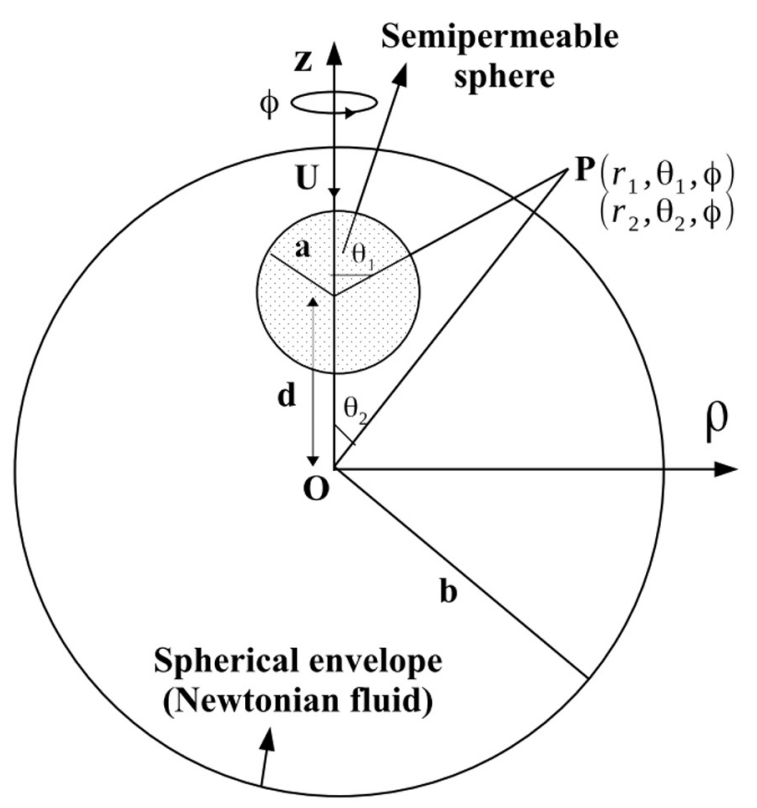

Fig. 1. Geometry of the problem 
For the flow field inside the semipermeable sphere, there are the continuity equation and the Darcy's law

$$
\begin{aligned}
\nabla \cdot \vec{v}^{(2)} & =0, \\
\vec{v}^{(2)} & =-\frac{k}{\mu} \nabla p^{(2)},
\end{aligned}
$$

where $\vec{v}^{(i)}$ and $p^{(i)}, i=1,2$ denote the velocity vector and the fluid pressure at any point, $\mu$ is the coefficient of viscosity, and $k$ is the permeability of the porous medium.

Since the considered problem is axially symmetric, all the flow quantities are independent of $\phi$. Thus, one can take the velocity vectors in the cylindrical coordinates as

$$
\vec{v}^{(i)}=v_{\rho}^{(i)} \vec{e}_{\rho}+v_{z}^{(i)} \vec{e}_{z}, \quad i=1,2
$$

Since $\nabla \cdot \vec{v}^{(i)}=0$, one can represent the velocity components $v_{\rho}^{(i)}$ and $v_{z}^{(i)}$ in terms of the Stokes stream function as

$$
v_{\rho}^{(i)}=\frac{1}{\rho} \frac{\partial \psi^{(i)}}{\partial z}, \quad v_{z}^{(i)}=-\frac{1}{\rho} \frac{\partial \psi^{(i)}}{\partial \rho}, \quad i=1,2
$$

The fourth order and second linear partial differential equations for the stream functions $\psi^{(i)}$, $i=1,2$ are obtained by eliminating the pressures from (2) and (4)

$$
\begin{aligned}
& E^{4} \psi^{(1)}=0 \\
& E^{2} \psi^{(2)}=0
\end{aligned}
$$

where $E^{2}=\frac{\partial^{2}}{\partial \rho^{2}}-\frac{1}{\rho} \frac{\partial}{\partial \rho}+\frac{\partial^{2}}{\partial z^{2}}$ is the Stokesian operator.

\section{Boundary conditions}

To determine the flow velocity components outside and inside the semipermeable sphere, continuity of pressure, normal velocity, zero tangential velocity at the interface are used, while at the cavity surface, continuity of radial velocity, Happel and Kuwabara boundary conditions are assumed $[9,12,13,17]$. Therefore, the following relations can be obtained

on $r_{1}=a$ :

$$
\begin{aligned}
v_{\rho}^{(1)} \tan \theta_{1}+v_{z}^{(1)} & =v_{\rho}^{(2)} \tan \theta_{1}+v_{z}^{(2)}, \\
v_{\rho}^{(1)} \cot \theta_{1}-v_{z}^{(1)} & =0, \\
p^{(1)} & =p^{(2)},
\end{aligned}
$$

on $r_{2}=b$ :

$$
v_{\rho}^{(1)} \tan \theta_{2}+v_{z}^{(1)}=-U
$$

(a) Happel model

$$
t_{r_{2} \theta_{2}}^{(1)}=0
$$

(b) Kuwabara model

$$
E^{2} \psi^{(1)}=0
$$




\section{Solution of the problem}

Using the principle of superposition, the stream functions $\psi^{(i)}$ of the fluid flow, which satisfy the boundary conditions on the spherical surfaces in the spherical coordinates $[2,4,5,8,10]$, are obtained by solving (7) and (8)

$$
\begin{aligned}
& \psi^{(1)}=\sum_{n=2}^{\infty}\left[\left(A_{n} r_{1}^{-n+1}+B_{n} r_{1}^{-n+3}\right) \vartheta_{n}\left(\xi_{1}\right)+\left(C_{n} r_{2}^{n}+D_{n} r_{2}^{n+2}\right) \vartheta_{n}\left(\xi_{2}\right)\right] \\
& \psi^{(2)}=\sum_{n=2}^{\infty}\left(e_{n} r_{1}^{n}+f_{n} r_{1}^{-n+1}\right) \vartheta_{n}\left(\xi_{1}\right) .
\end{aligned}
$$

The stream function $\psi^{(2)}$ is finite at the center of the semipermeable sphere and imposes the condition $f_{n}=0$. In (15) and (16), $\vartheta_{n}(\cdot)$ denotes the Gegenbauer function of the first kind of order $n$ and degree $-\frac{1}{2}$ and $\xi_{1}=\cos \theta_{1}, \xi_{2}=\cos \theta_{2}$. The unknown coefficients $A_{n}, B_{n}, C_{n}$, and $D_{n}$ can be determined using the boundary conditions (9)-(14).

The axial $v_{\rho}^{(i)}$ and radial $v_{z}^{(i)}$ velocity components, the pressure $p^{(i)}, i=1,2$, and the tangential stresses $t_{r_{2} \theta_{2}}^{(1)}$ are given by

$$
\begin{aligned}
v_{\rho}^{(1)} & =\sum_{n=2}^{\infty}\left[A_{n} \mathbf{A}_{1 n}\left(\zeta_{1}\right)+B_{n} \mathbf{B}_{1 n}\left(\zeta_{1}\right)+C_{n} \mathbf{C}_{1 n}\left(\zeta_{2}\right)+D_{n} \mathbf{D}_{1 n}\left(\zeta_{2}\right)\right], \\
v_{z}^{(1)} & =\sum_{n=2}^{\infty}\left[A_{n} \mathbf{A}_{2 n}\left(\zeta_{1}\right)+B_{n} \mathbf{B}_{2 n}\left(\zeta_{1}\right)+C_{n} \mathbf{C}_{2 n}\left(\zeta_{2}\right)+D_{n} \mathbf{D}_{2 n}\left(\zeta_{2}\right)\right], \\
p^{(1)} & =\sum_{n=2}^{\infty}\left[B_{n} \mathbf{B}_{3 n}\left(\zeta_{1}\right)+D_{n} \mathbf{D}_{3 n}\left(\zeta_{2}\right)\right], \\
t_{r_{2} \theta_{2}}^{(1)} & =\sum_{n=2}^{\infty}\left[A_{n} \mathbf{A}_{4 n}\left(\zeta_{1}\right)+B_{n} \mathbf{B}_{4 n}\left(\zeta_{1}\right)+C_{n} \mathbf{C}_{4 n}\left(\zeta_{2}\right)+D_{n} \mathbf{D}_{4 n}\left(\zeta_{2}\right)\right], \\
E^{2} \psi^{(1)} & =\sum_{n=2}^{\infty}\left[B_{n} \mathbf{B}_{7 n}\left(\zeta_{1}\right)+D_{n} \mathbf{D}_{7 n}\left(\zeta_{2}\right)\right] \\
v_{\rho}^{(2)} & =\sum_{n=2}^{\infty} E_{n} \mathbf{E}_{1 n}\left(\zeta_{1}\right), \\
v_{z}^{(2)} & =\sum_{n=2}^{\infty} E_{n} \mathbf{E}_{2 n}\left(\zeta_{1}\right), \\
p^{(2)} & =\sum_{n=2}^{\infty} E_{n} \mathbf{E}_{3 n}\left(\zeta_{1}\right),
\end{aligned}
$$

where $\zeta_{1}=\left(r_{1}, \theta_{1}\right), \zeta_{2}=\left(r_{2}, \theta_{2}\right)$ and

$$
\begin{array}{r}
\sum_{n=2}^{\infty}\left[A_{n} \mathbf{A}_{5 n}\left(\zeta_{3}\right)+B_{n} \mathbf{B}_{5 n}\left(\zeta_{3}\right)-E_{n} \mathbf{E}_{5 n}\left(\zeta_{3}\right)+C_{n} \mathbf{C}_{5 n}\left(\zeta_{4}\right)+D_{n} \mathbf{D}_{5 n}\left(\zeta_{4}\right)\right]=0 \\
\sum_{n=2}^{\infty}\left[A_{n} \mathbf{A}_{6 n}\left(\zeta_{3}\right)+B_{n} \mathbf{B}_{6 n}\left(\zeta_{3}\right)+C_{n} \mathbf{C}_{6 n}\left(\zeta_{4}\right)+D_{n} \mathbf{D}_{6 n}\left(\zeta_{4}\right)\right]=0 \\
\sum_{n=2}^{\infty}\left[B_{n} \mathbf{B}_{3 n}\left(\zeta_{3}\right)-E_{n} \mathbf{E}_{3 n}\left(\zeta_{3}\right)+D_{n} \mathbf{D}_{3 n}\left(\zeta_{4}\right)\right]=0
\end{array}
$$




$$
\begin{aligned}
\sum_{n=2}^{\infty}\left[A_{n} \mathbf{A}_{5 n}\left(\zeta_{5}\right)+B_{n} \mathbf{B}_{5 n}\left(\zeta_{5}\right)+C_{n} \mathbf{C}_{5 n}\left(\zeta_{6}\right)+D_{n} \mathbf{D}_{5 n}\left(\zeta_{6}\right)\right] & =-1, \\
\sum_{n=2}^{\infty}\left[A_{n} \mathbf{A}_{4 n}\left(\zeta_{5}\right)+B_{n} \mathbf{B}_{4 n}\left(\zeta_{5}\right)+C_{n} \mathbf{C}_{4 n}\left(\zeta_{6}\right)+D_{n} \mathbf{D}_{4 n}\left(\zeta_{6}\right)\right] & =0 \\
\sum_{n=2}^{\infty}\left[B_{n} \mathbf{B}_{7 n}\left(\zeta_{5}\right)+D_{n} \mathbf{D}_{7 n}\left(\zeta_{6}\right)\right] & =0
\end{aligned}
$$

where $\zeta_{3}=\left(1, \theta_{1}\right), \zeta_{4}=\left[\left(r_{2}, \theta_{2}\right)\right]_{r_{1}=1}, \zeta_{5}=\left[\left(r_{1}, \theta_{1}\right)\right]_{r_{1}=\frac{1}{\eta}}, \zeta_{6}=\left(\frac{1}{\eta}, \theta_{1}\right)$, and the functions $\mathbf{A}_{p n}$, $\mathbf{B}_{p n}, \mathbf{C}_{p n}, \mathbf{D}_{p n}$, and $\mathbf{E}_{p n}$ with $p=1, \ldots, 7$ are given in Appendix A.

\section{Drag on the semipermeable sphere}

The drag force can be calculated by using the formula

$$
F=\left.\pi \mu a \int_{0}^{\pi} r^{3} \sin ^{3} \theta \frac{\partial}{\partial r}\left(\frac{E^{2} \psi^{(1)}}{r^{2} \sin ^{2} \theta}\right) r\right|_{r=1} \mathrm{~d} \theta=-4 \pi \mu a U B_{2},
$$

which indicates that $B_{2}$ contributes to the hydrodynamic force experienced by the semipermeable sphere. The expression $B_{2}$ is the lowest order coefficient. The value of $B_{2}$ is the most precise and quickest convergent result.

The exact solution for the case of concentric semipermeable sphere, using the Happel and Kuwabara models, is given as

$$
\begin{aligned}
& F_{H p}=-4 \pi \mu a U\left[\frac{2\left(\beta^{2}-10\right) \eta^{5}+3 \beta^{2}}{2\left(\beta^{2}-10\right) \eta^{6}+3\left(2-\beta^{2}\right) \eta^{5}+\beta^{2}(3 \eta-2)-1}\right] \\
& F_{K u}=-4 \pi \mu a U\left[\frac{15 \beta^{2}}{2\left(\beta^{2}-10\right) \eta^{6}-10\left(2+\beta^{2}\right) \eta^{3}+2 \beta^{2}(9 \eta-5)-5}\right] .
\end{aligned}
$$

If there is no cavity surface, i.e., $a /(b-d)=0$, then the fluid is unbounded. The drag acting on a semipermeable sphere in a viscous fluid is given as [17]

$$
F_{\infty}=-4 \pi \mu a U\left[\frac{3 \beta^{2}}{2 \beta^{2}+1}\right]
$$

If $\beta \rightarrow \infty$, the drag exerted on a rigid sphere is obtained [8].

The wall correction factor $W$ is calculated as the ratio of the drag force acting on the semipermeable sphere in the closed boundary to the semipermeable sphere in the free medium. With the aid of (31) and (34), we get

$$
W=\frac{F}{F_{\infty}}
$$

\section{Results and discussion}

To obtain the numerical solution of the drag force exerted on a semipermeable sphere translating within a spherical cavity, let us choose boundary collocation points along the half-circular generating arcs of the semipermeable sphere and spherical cavity. The initial point $\theta_{i}=0$ and the terminal point $\theta_{i}=\pi$ are chosen along with the point $\theta_{i}=\pi / 2$ on both the arcs. If these 
three points are used in the system of equations (25)-(28) and in (29) and (30) for the Happel and Kuwabara models, respectively, then the coefficient matrix becomes a singular matrix. To avoid this difficulty and to achieve good accuracy, it is possible to use the method recommended in the literature $[1,2,4-6,10]$. The adjacent points of $\theta_{i}=0, \pi / 2, \pi$ are $\theta_{i}=\epsilon, \theta_{i}=\pi / 2-\epsilon$, $\theta_{i}=\pi / 2+\epsilon, \theta_{i}=\pi-\epsilon$, where $\epsilon$ is a specified value $\left(\epsilon=0.01^{\circ}\right)$ chosen so that the coefficient matrix is nonsingular. Let us divide the two quarter-circular arcs into equal segments so that additional points are selected as mirror-image pairs about $\theta=\pi / 2$. Choosing of finite number of discrete points on the arcs results in a system of linear equations. This system is solved by using the Gaussian elimination method.

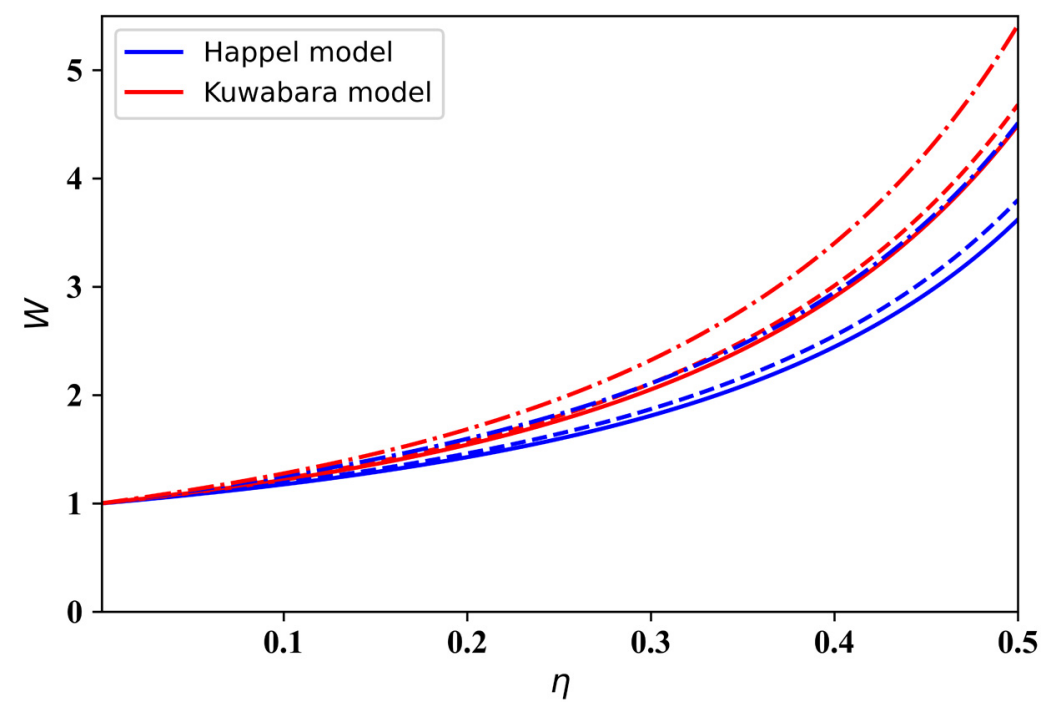

Fig. 2. Variation of $W$ versus $\eta$ for different values of $\delta$ (solid line $\delta \rightarrow 0$, dashed line $\delta=0.25$, and dash-dotted line $\delta=0.5)$ and fixed $k_{1}=0.001$

The numerical solution of the wall correction factor $W$ exerted on the semipermeable sphere in the nonconcentric cavity containing viscous fluid for both the aforementioned cases is presented in Fig. 2 and Tables 1 and 2. The obtained results converge to at least six decimal places. The following parameters are present in the problem:

Table 1. Convergence of the wall correction factor $W_{H p}$ for different values of $\delta, \eta$, and $k_{1}$

\begin{tabular}{|c|l|r|l|r|l|}
\hline \multicolumn{5}{|c|}{ wall correction factor $W_{H p}$ (Happel's model) } \\
\hline$N$ & $k_{1} \rightarrow 0$ & $N$ & $k_{1}=0.001$ & $N$ & $k_{1}=0.001$ \\
& $\eta=0.5$ & & $\eta=0.5$ & & $\eta=0.5$ \\
& $\delta=10^{-5}$ & & $\delta=0.25$ & & $\delta=0.5$ \\
\hline 6 & 3.62962 & 6 & 3.81408 & 6 & 4.78145 \\
8 & 3.62962 & 12 & 3.80314 & 12 & 4.54666 \\
& - & 18 & 3.80341 & 18 & 4.51816 \\
& - & 20 & 3.80343 & 24 & 4.51336 \\
& - & 22 & 3.80343 & 30 & 4.51310 \\
& - & & - & 36 & 4.51304 \\
& - & & - & 38 & 4.51303 \\
& - & & - & 40 & 4.51303 \\
& - & & - & & - \\
\hline
\end{tabular}


Table 2. Convergence of the wall correction factor $W_{K u}$ for different values of $\delta, \eta$, and $k_{1}$

\begin{tabular}{|c|l|r|l|r|l|}
\hline \multicolumn{6}{|c|}{ wall correction factor $W_{K u}$ (Kuwabara's model) } \\
\hline$N$ & $k_{1} \rightarrow 0$ & $N$ & $k_{1}=0.001$ & $N$ & $k_{1}=0.001$ \\
& $\eta=0.5$ & & $\eta=0.5$ & & $\eta=0.5$ \\
& $\delta=10^{-5}$ & & $\delta=0.25$ & & $\delta=0.5$ \\
\hline 6 & 4.50704 & 6 & 4.70113 & 6 & 5.93037 \\
8 & 4.50704 & 12 & 4.68211 & 12 & 5.43278 \\
& - & 18 & 4.68253 & 18 & 5.42203 \\
& - & 24 & 4.68251 & 24 & 5.41923 \\
& - & 30 & 4.68249 & 30 & 5.41850 \\
& - & 32 & 4.68249 & 36 & 5.41832 \\
& - & & - & 38 & 5.41828 \\
& - & & - & 40 & 5.41828 \\
\hline
\end{tabular}

Table 3. Wall correction factor $W_{H p}$ for different values of $k_{1}, \eta$, and $\delta$

\begin{tabular}{|c|c|c|c|c|}
\hline \multirow{2}{*}{$\delta$} & & \multicolumn{3}{|c|}{$W$} \\
\cline { 3 - 5 } & $\eta$ & $k_{1} \rightarrow 0$ & $k_{1}=0.001$ & $k_{1}=0.003$ \\
\hline 0.00001 & 0.001 & 1.00150 & 1.00150 & 1.00150 \\
& 0.010 & 1.01523 & 1.01522 & 1.01521 \\
& 0.100 & 1.17646 & 1.17636 & 1.17615 \\
& 0.200 & 1.42803 & 1.42772 & 1.42711 \\
& 0.300 & 1.81152 & 1.81076 & 1.80924 \\
& 0.400 & 2.44813 & 2.44615 & 2.44221 \\
& 0.500 & 3.62963 & 3.62346 & 3.61118 \\
\hline 0.25 & 0.001 & 1.00164 & 1.00164 & 1.00164 \\
& 0.010 & 1.01664 & 1.01663 & 1.01661 \\
& 0.100 & 1.19188 & 1.19177 & 1.19154 \\
& 0.200 & 1.46273 & 1.46238 & 1.46169 \\
& 0.300 & 1.87258 & 1.87170 & 1.86995 \\
& 0.400 & 2.55082 & 2.54849 & 2.54386 \\
& 0.500 & 3.81083 & 3.80343 & 3.78873 \\
\hline 0.5 & 0.001 & 1.00219 & 1.00219 & 1.00218 \\
& 0.010 & 1.02215 & 1.02214 & 1.02212 \\
& 0.100 & 1.25230 & 1.25214 & 1.25181 \\
& 0.200 & 1.59805 & 1.59753 & 1.59648 \\
& 0.300 & 2.11016 & 2.10875 & 2.10592 \\
& 0.400 & 2.95211 & 2.94811 & 2.94014 \\
& 0.500 & 4.52649 & 4.51302 & 4.48640 \\
\hline
\end{tabular}

1. The separation parameter: $\eta=a / b(0<\eta<0.5)$.

2. The parameter $\delta=\frac{d}{b-a}$ is the normalized deviation distance of the center of inner sphere from the center of spherical container: If $\delta \rightarrow 0$, the center of inner sphere and spherical container coincide and the problem is reduced to translation of a semipermeable sphere in a concentric spherical container. 
Table 4. Wall correction factor $W_{K u}$ for different values of $k_{1}, \eta$, and $\delta$

\begin{tabular}{|c|c|c|c|c|}
\hline \multirow{2}{*}{$\delta$} & & \multicolumn{3}{|c|}{$W$} \\
\cline { 3 - 5 } & $\eta$ & $k_{1} \rightarrow 0$ & $k_{1}=0.001$ & $k_{1}=0.003$ \\
\hline 0.00001 & 0.001 & 1.00180 & 1.00180 & 1.00180 \\
& 0.010 & 1.01833 & 1.01832 & 1.01830 \\
& 0.100 & 1.21803 & 1.21789 & 1.21762 \\
& 0.200 & 1.54324 & 1.54278 & 1.54187 \\
& 0.300 & 2.05400 & 2.05269 & 2.05007 \\
& 0.400 & 2.91392 & 2.90998 & 2.90215 \\
& 0.500 & 4.50704 & 4.49347 & 4.46662 \\
\hline 0.25 & 0.001 & 1.00191 & 1.00191 & 1.00190 \\
& 0.010 & 1.01937 & 1.01936 & 1.01934 \\
& 0.100 & 1.23013 & 1.22998 & 1.22969 \\
& 0.200 & 1.57255 & 1.57206 & 1.57108 \\
& 0.300 & 2.10992 & 2.10850 & 2.10566 \\
& 0.400 & 3.01556 & 3.01127 & 3.00274 \\
& 0.500 & 4.69741 & 4.68248 & 4.65293 \\
\hline 0.5 & 0.001 & 1.00232 & 1.00231 & 1.00231 \\
& 0.010 & 1.02352 & 1.02351 & 1.02349 \\
& 0.100 & 1.27753 & 1.27734 & 1.27698 \\
& 0.200 & 1.68611 & 1.68548 & 1.68420 \\
& 0.300 & 2.32583 & 2.32397 & 2.32025 \\
& 0.400 & 3.40895 & 3.40327 & 3.39192 \\
& 0.500 & 5.43868 & 5.41828 & 5.37759 \\
\hline
\end{tabular}

3. The permeability parameter $\beta=a / \sqrt{k}$ : If $\beta \rightarrow \infty$, the special case of translation of a solid sphere in an eccentric spherical container is obtained.

Fig. 2 depicts the variation of the wall correction factor $W$ with the separation parameter $\eta$ for different values of $\delta$ and for the fixed value of the permeability parameter $k_{1}(=1 / \beta=$ $\sqrt{k} / a$ ). It is observed that $W$ is a monotonic increasing function of $\eta$. Tables 1 and 2 indicate that $W$ decreases with an increase in nonzero permeability parameter $k_{1}$ and increases with an increase in the separation parameter $\eta$. It is found that the drag force on an eccentric semipermeable sphere is less than the drag force exerted on an eccentric solid sphere in a bounded spherical medium. Collocation results of $W$ in Table 1 for the concentric case $(\delta \rightarrow 0)$ agree with the exact solutions and for a nonconcentric particle $(\delta \neq 0)$ of solid case, $k_{1} \rightarrow 0$ matches with the results of Faltas and Saad [2].

\section{Conclusions}

In this work, the numerical solution for the slow motion of a semipermeable sphere in a nonconcentric spherical cavity was studied. The wall correction factor acting on the particle was calculated for different parameter values and the results showed that the solution procedure converges rapidly. It was observed that the wall correction factor is an increasing function of separation parameter and is a decreasing function of the permeability parameter. The distance between the centers of the particle and the container played a significant role in evaluating 
the values of the wall correction factor. It was noted that the wall correction factor is minimal when the particle is at concentric position and increases monotonically with the relative distance between the centers of the particle and the container. Also, it was found that the drag force on an eccentric semipermeable sphere is less than the drag force exerted on an eccentric solid sphere in a bounded spherical medium.

\section{References}

[1] Dagan, Z., Weinbaum, S., Pfeffer, R., General theory for the creeping motion of a finite sphere along the axis of a circular orifice, Journal of Fluid Mechanics 117 (1982) 143-170. https://doi.org/10.1017/S0022112082001566

[2] Faltas, M. S., Saad, E. I., Stokes flow past an assemblage of slip eccentric spherical particle-in-cell models, Mathematical Methods in Applied Sciences 34 (2011) 1594-1605. https://doi.org/10.1002/mma.1465

[3] Feng, Z. G., Michaelides, E. E., Motion of a permeable sphere at finite but small Reynolds numbers, Physics of Fluids 10 (6) (1998) 1375-1383. https://doi.org/10.1063/1.869662

[4] Ganatos, P., Weinbaum, S., Pfeffer, R., A strong interaction theory for the creeping motion of a sphere between plane parallel boundaries. Part 1. Perpendicular motion, Journal of Fluid Mechanics 99 (1980) 739-753. https://doi.org/10.1017/S0022112080000870

[5] Ganatos, P., Weinbaum, S., Pfeffer, R., A strong interaction theory for the creeping motion of a sphere between plane parallel boundaries. Part 2. Parallel motion, Journal of Fluid Mechanics 99 (1980) 755-783. https://doi.org/10.1017/S0022112080000882

[6] Gluckman, M.J., Pfeffer, R., Weinbaum, S., A new technique for treating multiparticle slow viscous flow: Axisymmetric flow past spheres and spheroids, Journal of Fluid Mechanics 50 (1971) 705-740. https://doi.org/10.1017/S0022112071002854

[7] Happel, J., Viscous flow in multiparticle systems: Slow motion of fluids relative to beds of spherical particles, American Institute of Chemical Engineers Journal 4 (1958) 197-201. https://doi.org/10.1002/aic.690040214

[8] Happel, J., Brenner, H., Low Reynolds number hydrodynamics with special applications to particulate media, Englewood Cliffs, Prentice-Hall, 1965.

[9] Joseph, D.D., Tao, L.N., The effect of permeability in the slow motion of a porous sphere in a viscous liquid, Journal of Applied Mathematics and Mechanics 44 (1964) 361-364. https://doi.org/10.1002/zamm.19640440804

[10] Keh, H. J., Lee, T.C., Axisymmetric creeping motion of a slip spherical particle in a nonconcentric spherical cavity, Theoretical and Computational Fluid Dynamics 24 (2010) 497-510. https://doi.org/10.1007/s00162-010-0181-y

[11] Khanukaeva, D. Yu., Filippov, A. N., Yadav, P. K., Tiwari, A., Creeping flow of micropolar fluid parallel to the axis of cylindrical cells with porous layer, European Journal of Mechanics-B/Fluids 76 (2019) 73-80. https://doi.org/10.1016/j.euromechflu.2019.01.012

[12] Krishna Prasad, M., Cell models for non-Newtonian fluid past a semipermeable sphere, International Journal of Mathematical, Engineering and Management Sciences 4 (6) (2019) 1352-1361. https://doi.org/10.33889/IJMEMS.2019.4.6-106

[13] Krishna Prasad, M., Bucha, T., Effect of magnetic field on the steady viscous fluid flow around a semipermeable spherical particle, International Journal of Applied and Computational Mathematics 5 (2019) No. 98. https://doi.org/10.1007/s40819-019-0668-1

[14] Kuwabara, S., The forces experienced by randomly distributed parallel circular cylinders or spheres in a viscous flow at small Reynolds numbers, Journal of the Physical Society of Japan 14 (1959) 527-532. https://doi.org/10.1143/JPSJ.14.527 
[15] Leichtberg, S., Pfeffer, R., Weinbaum, S., Stokes flow past finite coaxial clusters of spheres in a circular cylinder, International Journal Multiphase Flow 3 (1976) 147-169. https://doi.org/10.1016/0301-9322(76)90005-7

[16] Leonov, A. I., The slow stationary flow of a viscous fluid about a porous sphere, Journal of Applied Mathematics and Mechanics 26 (3) (1962) 842-847. https://doi.org/10.1016/0021-8928(62)90050-3

[17] Shapovalov, V. M., Viscous fluid flow around a semipermeable particle, Journal of Applied Mechanics and Technical Physics 50 (4) (2009) 584-588. https://doi.org/10.1007/s10808-009-0079-x

[18] Srinivasacharya, D., Flow past a porous approximate spherical shell, Journal of Applied Mathematics and Physics, 58 (2007) 646-658. https://doi.org/10.1007/s00033-006-6003-9

[19] Srivastava, B. G., Yadav, P. K., Deo, S., Singh, P. K., Filippov, A., Hydrodynamic permeability of a membrane composed of porous spherical particles in the presence of uniform magnetic field, Colloid Journal, 76 (6) (2014) 725-738. https://doi.org/10.1134/S1061933X14060167

[20] Vereshchagin, A.S., Dolgushev, S. V., Low-velocity viscous incompressible fluid flow around a hollow porous sphere, Journal of Applied Mechanics and Technical Physics, 52 (3) (2011) 406-414. https://doi.org/10.1134/S0021894411030114

[21] Yadav, P. K., Deo, S., Yadav, M. K., Filippov, A., On hydrodynamic permeability of a membrane built up by porous deformed spheroidal particles, Colloid Journal 75 (5) (2013) 611-622. https://doi.org/10.1134/S1061933X13050165

[22] Yadav, P. K., Tiwari, A., Deo, S., Filippov, A.N., Vasin, S. I., Hydrodynamic permeability of membranes built up by spherical particles covered by porous shells: Effect of stress jump condition, Acta Mechanica, 215 (2010) 193-209. https://doi.org/10.1007/s00707-010-0331-8

[23] Yadav, P.K., Tiwari, A., Singh, P., Hydrodynamic permeability of a membrane built up by spheroidal particles covered by porous layer, Acta Mechanica, 229 (2018) 1869-1892. https://doi.org/10.1007/s00707-017-2054-6

[24] Yadav, P. K., Tiwari, A., Singh, P., Motion through spherical droplet with non-homogenous porous layer in spherical container, Applied Mathematics and Mechanics 41 (7) (2020) 1069-1082. https://doi.org/10.1007/s10483-020-2628-8 


\section{Appendix A}

The functions appearing in (25)-(30) are defined as

$$
\begin{aligned}
& \mathbf{A}_{1 n}(\zeta)=-r^{-n-1}(n+1) \vartheta_{n+1}(\xi)\left(1-\xi^{2}\right)^{-1 / 2}, \\
& \mathbf{B}_{1 n}(\zeta)=-r^{-n+1}\left[(n+1) \vartheta_{n+1}(\xi)-2 \xi \vartheta_{n}(\xi)\right]\left(1-\xi^{2}\right)^{-1 / 2}, \\
& \mathbf{C}_{1 n}(\zeta)=-r^{n-2}\left[(n+1) \vartheta_{n+1}(\xi)-(2 n-1) \xi \vartheta_{n}(\xi)\right]\left(1-\xi^{2}\right)^{-1 / 2} \\
& =\mathbf{E}_{1 n}(\zeta) \text {, } \\
& \mathbf{D}_{1 n}(\zeta)=-r^{n}\left[(n+1) \vartheta_{n+1}(\xi)-(2 n+1) \xi \vartheta_{n}(\xi)\right]\left(1-\xi^{2}\right)^{-1 / 2} \text {, } \\
& \mathbf{A}_{2 n}(\zeta)=-r^{-n-1} P_{n}(\xi), \\
& \mathbf{B}_{2 n}(\zeta)=-r^{-n+1}\left[2 \vartheta_{n}(\xi)+P_{n}(\xi)\right], \\
& \mathbf{C}_{2 n}(\zeta)=-r^{n-2}\left[(2 n-1) \vartheta_{n}(\xi)+P_{n}(\xi)\right] \\
& =\mathbf{E}_{2 n}(\zeta) \text {, } \\
& \mathbf{D}_{2 n}(\zeta)=-r^{n}\left[(2 n+1) \vartheta_{n}(\xi)+P_{n}(\xi)\right] \text {, } \\
& \mathbf{B}_{3 n}(\zeta)=-\frac{2(2 n-3)}{n} r^{-n} P_{n-1}(\xi), \\
& \mathbf{D}_{3 n}(\zeta)=-\frac{2(2 n+1)}{(n-1)} r^{n-1} P_{n-1}(\xi), \\
& \mathbf{E}_{3 n}(\zeta)=\beta^{2} \frac{1}{(n-1)} r^{n-1} P_{n-1}(\xi), \\
& \mathbf{A}_{4 n}(\zeta)=2\left(n^{2}-1\right) r^{-n-2} \vartheta_{n}(\xi)\left(1-\xi^{2}\right)^{-1 / 2}, \\
& \mathbf{B}_{4 n}(\zeta)=2 n(n-2) r^{-n} \vartheta_{n}(\xi)\left(1-\xi^{2}\right)^{-1 / 2}, \\
& \mathbf{C}_{4 n}(\zeta)=2 n(n-2) r^{n-3} \vartheta_{n}(\xi)\left(1-\xi^{2}\right)^{-1 / 2} \text {, } \\
& =\mathbf{E}_{4 n}(\zeta) \text {, } \\
& \mathbf{D}_{4 n}(\zeta)=2\left(n^{2}-1\right) r^{n-1} \vartheta_{n}(\xi)\left(1-\xi^{2}\right)^{-1 / 2}, \\
& \mathbf{A}_{5 n}(\zeta)=\mathbf{A}_{1 n}(\xi) \xi^{-1}\left(1-\xi^{2}\right)^{1 / 2}+\mathbf{A}_{2 n}(\xi), \\
& \mathbf{B}_{5 n}(\zeta)=\mathbf{B}_{1 n}(\xi) \xi^{-1}\left(1-\xi^{2}\right)^{1 / 2}+\mathbf{B}_{2 n}(\xi), \\
& \mathbf{C}_{5 n}(\zeta)=\mathbf{C}_{1 n}(\xi) \xi^{-1}\left(1-\xi^{2}\right)^{1 / 2}+\mathbf{C}_{2 n}(\xi), \\
& \mathbf{D}_{5 n}(\zeta)=\mathbf{D}_{1 n}(\xi) \xi^{-1}\left(1-\xi^{2}\right)^{1 / 2}+\mathbf{D}_{2 n}(\xi) \text {, } \\
& \mathbf{A}_{6 n}(\zeta)=\mathbf{A}_{1 n}(\xi) \xi\left(1-\xi^{2}\right)^{-1 / 2}-\mathbf{A}_{2 n}(\xi), \\
& \mathbf{B}_{6 n}(\zeta)=\mathbf{B}_{1 n}(\xi) \xi\left(1-\xi^{2}\right)^{-1 / 2}-\mathbf{B}_{2 n}(\xi), \\
& \mathbf{C}_{6 n}(\zeta)=\mathbf{C}_{1 n}(\xi) \xi\left(1-\xi^{2}\right)^{-1 / 2}-\mathbf{C}_{2 n}(\xi), \\
& \mathbf{D}_{6 n}(\zeta)=\mathbf{D}_{1 n}(\xi) \xi\left(1-\xi^{2}\right)^{-1 / 2}-\mathbf{D}_{2 n}(\xi) \text {, } \\
& \mathbf{B}_{7 n}(\zeta)=-2(2 n-3) r^{-n+1} \vartheta_{n}(\xi), \\
& \mathbf{D}_{7 n}(\zeta)=2(2 n+1) r^{n} \vartheta_{n}(\xi) \text {. }
\end{aligned}
$$


M. Krishna Prasad / Applied and Computational Mechanics 15 (2021) 19-30

Additionally, the article utilises the following relations between the spherical $(r, \theta, \phi)$ and cylindrical $(\rho, \phi, z)$ coordinate systems:

$$
\begin{array}{ll}
r_{1}=\left[\rho^{2}+(z-d)^{2}\right]^{1 / 2}, \cos \theta_{1}=\frac{z-d}{r_{1}}, \sin \theta_{1}=\frac{\rho}{r_{1}}, \\
r_{2}=\left[\rho^{2}+z^{2}\right]^{1 / 2}, \quad \cos \theta_{2}=\frac{z}{r_{2}},
\end{array}
$$

